\title{
Course Reform in Senior Undergraduate Teaching: a Case Study in China
}

\author{
Zhe Chen ${ }^{1 *}$, Xin Peng ${ }^{1}$ and Yichuan Pan $^{1}$ \\ ${ }^{1}$ Shandong Jiaotong University, Jinan 250357, China; \\ * Corresponding author
}

Keywords: course reform; self-directed learning; problem-based learning; problem cooperating.

\begin{abstract}
Problem-based learning has been widely applied in a number of professional curricula. Implementations indicate that problem-based learning is valid and effective in several domains; problem-solving ability and self-directed learning ability of the students are greatly enhanced in problem-based learning programs. From 2013, problem-based learning was applied in the course "Shape Design of Yacht” in Shandong Jiaotong University as a part of course reform in the discipline of naval architecture and marine engineering. Group work and self-directed learning are encouraged in class to resolve pre-set problems. Meanwhile, a 3-index assessment system established to match Problem-based learning. Results of our course evaluations show that $91 \%$ of the students found the new approach to be a helpful, useful, and interesting way in this course, nevertheless, some optimizations such as grouping method in problem-based learning still need be done in the future.
\end{abstract}

\section{Introduction}

For a long time, the university phase was recognized as a transition between a student and a worker. In this special medium period, some skills such as teamwork, problem-solving ability and self-directed learning ability are significant for undergraduates. In traditional lecture-based learning (LBL) approach, the teacher is the lecture leader, standing in front of the class, with endless speak for fifty minutes or longer. Meanwhile, the student, whose responsibility is to acquire knowledge, is expected to concentrate on what is absolutely new for them for a whole lecture. However, new knowledge does not mean it is fresh and interesting. Instead, most of the knowledge is boring and not easy to understand. In many situations, as the youngsters can hardly pay attention to the teachers' words all through the class, LBL is not an effective teaching method.

Nowadays, various teaching instruments and learning approaches are applied in universities; the student is encouraged to study with these supports. Problem-based learning (PBL), as a student-centred learning approach, was firstly implemented in McMaster University (1969) to encourage life-long, self-directed learning for the study of medicine. After a long term development, the PBL approach become widespread in economics, law and psychology[1].

\section{PBL Approach and Literature Review}

Although PBL approach was implemented in a number of disciplines, and there are eight different modes of implementing PBL available to educationalists[2]. The foundation of PBL is mostly Barrows' theory. According to Barrows' theory, PBL is divided into three phases in practice. The first phase can be defined as a problem encounter; the unfamiliar problem was encountered to students at this stage. Subsequently, at problem cooperating phase, the students will be divided into several small groups to identify the problem. Then the self-directed learning phase, each student learns the knowledge based on the identified problem individually. At last, the process ends with another problem cooperating phase, students solve the problem with cooperative work. PBL approach encourages students the leadership, more activity in course learning participation.

In the PBL approach, before the course started, problems should be properly prepared. Once a lecture is started, students are organized into 5-6 person groups, which are leading the whole process. In cooperating phase, teachers and tutors are supposed to play roles of facilitators, they motivate and assist students to identify what information is already known on the problem, which information they need to know to solve it 
[3]. Group cooperating (teamwork) and self-directed learning of the students are emphasized here, the knowledge they learned are applied immediately in the problem-solving process[4]. Hence, problem-solving skills are improved. With the implement of PBL, the students are encouraged to become life-long, independent learners. There are three main outcomes of PBL:

1)acquisition of factual knowledge; 2)mastery of general principles or concepts that can be transferred to solve similar problems; and 3)acquisition of prior examples that can be used in future situations of a similar nature[5, 6].

PBL application in engineering education has been applied both in fundamental and specific subjects, from mathematics to engineering application program[7, 8]. PBL in engineering education utilizes often a badly structured real-life situation that is tackled as a project. In this situation, necessary adjustments are required. There are some adjustments on PBL in specific applications, such as 'Problem-Oriented and Project-Based Learning' and 'Problem-Based Project-Organized Learning' by learning around problem scenarios[9].

Most applications in PBL are successful in implementation. Rui M. Lima etc. investigated 367 participants by questionnaire from 16 institutions in Brazil for 5 years. Vialed respondents showed that 95\% of students supported that workshops had an impact on their teaching practice, and more than $70 \%$ of students would like to keep on participating in PBL lectures[10]. Practices in other countries also presented tremendous successes.

Since the 1990s, PBL began to apply in China in health science, such as medicine, dentistry and nursing. Before the 2000s, the teaching resources were not well spread, and the teachers were not familiar with this new approach, PBL only implemented in a limited number of universities [11].

Experiment instrument and supported workshop developed later, PBL application became popular in various universities and institutes. Cooperative practice between Beijing Normal University (China) and Aalborg University (Denmark) indicated a successful application in interdisciplinary research [12]. Nowadays, More assist devices and methods such as Wechat (a multipurpose social communication software in China), Massive open online course (MOOC) and case-based learning (CBL) are embedded in the PBL approach.

\section{A PBL Practice Applied in Shandong Jiaotong University (SDJTU)}

About six years ago, the naval architecture and marine engineering discipline of SDJTU launched a new strategic plan to match the university's position in the plication-oriented institution. In order to enhance the association between theory and practice, besides knowledge, skills and attitudes were also under close scrutiny from the school. Conceiving, designing, implementing and operating (CDIO) was released to encourage students to master creative and operational skills as well as deeper working knowledge[1]. Major curriculum reform was conducted as the plan required.

From 2013, PBL was implemented for the postgraduate and senior undergraduate students in the discipline of naval architecture and marine engineering in SDJTU. Newly built and re-modelled workshops and labs properly supported the PBL approach.

Table 1 Comparison of course menu SDOY

\begin{tabular}{ccl}
\hline Course menu of LBL class & Course menu of PBL class \\
\hline - The principle of shape and shape design & - & Definition of yacht parameters \\
- The procedure of shape design of a yacht & - & The concept of the shape \\
- Inspection, material, manufacturing, etc. & - Sketch and optimization \\
- effect in shape design & - & - \\
Yacht shape design practice & - Modelling and rendering \\
\hline
\end{tabular}

One implementation on PBL is "Shape Design of Yacht (SDOY)". The course is an obligatory course for senior students learning yacht design and manufacturing. Its content consists of the theory of shape design, process and practice of yacht design. Practice experience in estimating some related elements, such as mechanical, mechanics, material and manufacturing is also included. We used to apply LBL approach in 
SDOY course, the course menu is shown as the left column of Table 1. According to this order, the teacher taught all the related principles and theories. After that, a related practice was at the end of the curriculum. After a reform on PBL is applied, new course menu was established, shows as the right column of Table 1. Compared to the course menu in LBL, the principles and theories were eliminated, and the new menu was set up according to the procedure of yacht shape design.

In PBL approach, the course is defined as a big task or a huge case, 'shape design of a yacht'. Then the case is split into various phases (chapters), shown as the menu on the right column in Table 1.

Metacognition is the process of knowing how one knows or learns[8]. Before the lecture starts, all the related knowledge is refined and formed into a lot of metacognition, and then the teacher proposes the problem according to the metacognition. In this course, each problem is not only an independent case but also a component of the big task. There is a summary of 10 problems in the course shown in table 2, whose solutions cover all the contents of the principles and theories in SDOY.

Table 2 Problems of each chapter

\begin{tabular}{lll}
\hline \multicolumn{1}{c}{ Chapter } & \multicolumn{1}{c}{ Problem } \\
\hline \multirow{2}{*}{ Definition of yacht parameters } & - What is the requirement of the shipowner? \\
\hline The concept of the shape & - How to define the dimension of the boat? \\
\hline Sketch and optimization & - What is the main concept of the design? \\
\hline $3-d$ views and layout drawing & - What is the foundation of your optimization? \\
\hline \multirow{2}{*}{ Modelling and rendering } & inspection/ balance/ maintenance/ economic? \\
\hline
\end{tabular}
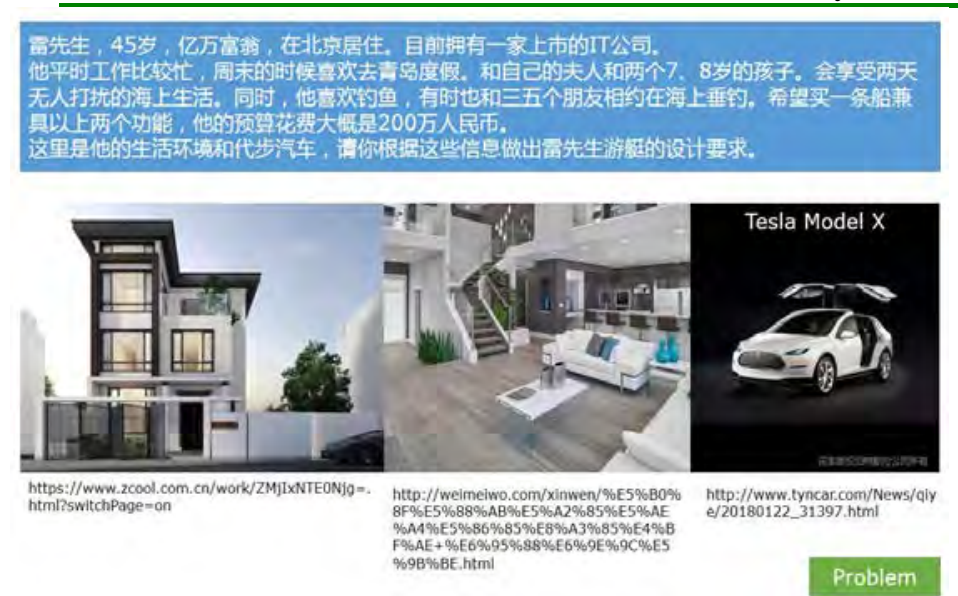

(Translation of the words in the form left: $\mathrm{Mr}$ Lei, 45, a billionaire, lives in Beijing. Currently operates an IT company. He is very busy on workdays. On weekends, he likes to travel to Qingdao on vacation with his wife and two children, 7 and 8 years old respectively, enjoying two days of undisturbed sea life. Meanwhile, fish is one of his hobbies, and sometimes he would love to fish at sea with several friends. He hopes to buy a boat with these two usages. His budget is about $¥ 2,000,000$. There are his living environment and vehicle condition, please describe the design requirements of the motor yacht.)

Fig. 1 A slide page from chapter 1 which illustrated a problem

The first problem on the problem list in Table 2 is selected as an instance, the problem is proposed in a slide page as Fig. 1 shows. One week before the class starts, the problem is revealed to the students from the course information platform (CIP) as well as some suggested books and knowledge. Then students are divided into 5-6 person groups. Each group has a manager, in charge of all the issues of the group in course learning.

Applying CDIO, group members cooperate on the complex design problems shown in the slides. In the discussion, the students communicate on existing knowledge; try to find feasible solutions to the problem. The discussion often cost 30 minutes. The teacher participates as a guide. Because only the teacher has information about the learning goals, his participant can bring some advice and guidance in the discussion. Moreover, the teacher can also clarify these concepts in class and bring the students up to speed. In this specific problem, the teacher suggests the students pay more attention to the character description and picture 
illustration. Related knowledge then reveals to the students after the discussion. Subsequent, students need to learn the knowledge themselves after class. Self-directed learning based on the problem is encouraged. The next class, students discuss the problem again and resolve it. Finally, one student in the group needs to make a presentation on the problem-solving process, group members take turns to represent. As a conclusion, the teacher analyzes the problem and reviews the presentation of each group in problem-solving. In this course learning, the same circuit of problem-solving is conducted, again and again, ended after all the problems are solved. Sometimes, groups do not follow the teacher's clue, get a correct but unexpected conclusion, they need to be prized as who get an expected answer.

As a part of PBL reform in the course, 3-index assessment system is set up. The final exam is substituted by a report. Two weeks after the course completed, each group need to hand in a report. The report should cover a new design and all the problems with the corresponding solution. Process evaluation is also valued in teaching; the teacher evaluates the performance in discussion and presentation of each student in class. Design report, teamwork performance and personal presentation; construct the 3-index assessment system.

\section{Assessment of PBL Implement in SDJTU}

In order to evaluate the implementation of PBL properly, a mixed assessment was applied every year. The assessment includes two parts, questionnaire investigation and the face to face interview.

Firstly, a questionnaire investigation was applied. The survey was conducted after the students submitted their reports. In 4 years, 154 students were suggested to participate in the survey, and 134 valid questionnaires were collected. Results show that respondents thought new method an effective way of learning theory and applications of yacht shape design. 91\% respondents believed PBL is a helpful, useful, and more interesting way in course learning, most of them considered problems in this course were set properly, and their self-directed learning ability improved during the problem-solving process. 93\% of respondents preferred PBL to LBL, while 72\% participates thought the relationship between student and teacher was closer in PBL course. 90\% respondents thought they were more active in current PBL course; they concentrated on how to solve problems and enjoy the solving process during course learning. As a substitute for paper examination, according to the survey, presentation and course report were also preferred by the students. $74 \%$ respondents thought it a promotion in applying complex knowledge; while $88 \%$ participates believed new assessment method avoided boring reciting period before the examination. In the recent three years, as a support, CIP was applied in SDJTU in most courses. By using the CIP, problems and assignments could be arranged promptly. In the survey these years, CIP in PBL was also listed in questionnaires, 113 students joined this course and 99 valid questionnaires were submitted. Most of them (91\%) believed CIP is helpful for PBL. The investigation seems to illustrate an excellent conclusion because very few respondents proposed a suggestion for PBL. To make a further assessment on PBL in the questionnaire investigation, face to face interview was applied in the last three years.

The object of the interview is also selected from the students who joined the course. At the end of the course semester, 5 students are selected to join the interview, from the highest to the lowest, one in every $20 \%$ segment score group. The modus of the interview is one-on-one, and the duration is about 15 minutes. The students were just asked to talk about the positive and negative of PBL in SDOY. As we expected, some negatives revealed after the interviews. Normally, the small group in course learning was constructed in a random way; it omitted the learning conditions of prerequisite courses, which meant that the pre-cognition level of each student was not balanced. Some students might find it difficult to follow others in teamwork. Others considered self-directed learning occupied more spare time, it increased their learning burdens.

Since last year, an investigation for graduated students on course arrangement started, most of them thought PBL approach was a good attempt, enlarged their knowledge, transformed their learning mode, enhanced their confidence, and brought benefit to their current work. 


\section{Conclusion}

PBL approach, as a successful practice, implemented in various areas in course learning. It integrated across the senior students SDOY learning at SDJTU. The component changed in PBL course and 10 problems were set for the learning process.

Through assessment activities, PBL is an effective way of course teaching. Most students thought it could bring up teamwork, self-directed learning, useful and helpful in their life-long study. However, the current grouping method may obstacle some students' activities. Next stage, the grouping method need to be optimized in the future by analyzing the performance of each student in prerequisite courses.

\section{Acknowledgement}

This work was financially supported by the Postgraduate educational grant of SDJTU (2017YJ001, 2017YJ008).

\section{References}

[1]. K. Edström, and A. Kolmos, PBL and CDIO: complementary models for engineering education development. European Journal of Engineering Education, 2014. 39(5): pp. 539-555.

[2]. M. Savin-Baden. Problem-based learning: Reason in madness. in Proceedings of problem-based learning conference 2004: A quality experience-across the boundaries, across the disciplines, across the globe. 2006.

[3]. S.E. Sikkema, and J.A. Sauerwein, Exploring culture-specific learning styles in accounting education. Journal of International Education in Business, 2015. 8(2): pp. 78-91.

[4]. H.S. Barrows, A taxonomy of problem - based learning methods. Medical education, 1986. 20(6): pp. 481-486.

[5]. A.M. Hasna. Problem based learning in engineering design. In Proceedings of SEFI 36TH Annual Conference, European Society for Engineering Education. 2008.

[6]. G. Tortorella, and P.A. Cauchick-Miguel, Teaching lean manufacturing at a postgraduate level: Integrating traditional teaching methods and problem-based learning approach. International Journal of Lean Six Sigma, 2018. 9(3): pp. 301-323.

[7]. J. Perrenet, P. Bouhuijs, and J. Smits, The suitability of problem-based learning for engineering education: theory and practice. Teaching in higher education, 2000. 5(3): pp. 345-358.

[8]. D.R. Brodeur, P.W. Young, and K.B. Blair. Problem-based learning in aerospace engineering education. in Proceedings of the 2002 American Society for Engineering Education Annual Conference and Exposition, Montreal, Canada. 2002.

[9]. A. Al Mughrabi, and M. Jaeger, Utilising a capability maturity model to optimise project based learning-case study. European Journal of Engineering Education, 2018. 43(5): pp. 679-692.

[10]. R.M. Lima, D. Mesquita, and L. Coelho, Five years of project-based learning training experiences in higher education institutions in Brazil. 2017.

[11]. G. Song, et al., Perspectives: Exploratory thoughts concerning educational reform with problem-based learning in China. Teaching and Learning in Medicine, 2005. 17(4): pp. 382-384.

[12]. X. Du, L. Su, and J. Liu, Developing sustainability curricula using the PBL method in a Chinese context. Journal of Cleaner Production, 2013. 61: pp. 80-88. 